\title{
LINEAR RELATIONS BETWEEN HIGHER ADDITIVE COMMUTATORS
}

\author{
O. TAUSSKY AND H. WIELANDT ${ }^{1}$
}

It has been noticed earlier [1] that the iterated commutators

$$
b_{1}=a b-b a, \quad b_{i}=a b_{i-1}-b_{i-1} a \quad(i=2,3, \cdots)
$$

of $2 \times 2$ matrices $a, b$ with elements in a field $F$ (which we may assume algebraically closed) satisfy the linear relation

$$
b_{3}=\left(\alpha_{1}-\alpha_{2}\right)^{2} b_{1}
$$

where $\alpha_{1}, \alpha_{2}$ denote the eigenvalues of $a$. Although it is easily seen and well known (cf. e.g. [2]) that also for any two $n \times n$ matrices $a, b$, where $n>2$, there exist linear relations between the $b_{i}$ whose coefficients are independent of $b$ no such relation seems to be explicitly known. We want to generalize (2) to $n \times n$ matrices.

The mapping $x \rightarrow A x:=a x-x a$ is a linear operator on the $n^{2}$ dimensional space $F_{n \times n}$ of all $n \times n$ matrices $x$ with elements in $F$. By the Hamilton-Cayley Theorem $A$ satisfies its characteristic equation, say $h(A)=0$. If $\alpha_{1}, \cdots, \alpha_{n}$ denote the eigenvalues of $a$ the eigenvalues of $A$ are known [3] to be $\alpha_{i}-\alpha_{j}(i, j=1,2, \cdots, n)$, hence

$$
\begin{aligned}
h(z) & =\prod_{i, j}\left[z-\left(\alpha_{i}-\alpha_{j}\right)\right]=z^{n} \prod_{i<j}\left[z^{2}-\left(\alpha_{i}-\alpha_{j}\right)^{2}\right] \\
& =z^{n} \cdot\left(z^{2 N}-\delta_{1} z^{2 N-2}+\delta_{2} z^{2 N-4}-\cdots+(-1)^{N} \delta_{n}\right)
\end{aligned}
$$

where $N=n(n-1) / 2$ and $\delta_{k}$ is the $k$ th elementary symmetric function of the $\left(\alpha_{i}-\alpha_{j}\right)^{2}, 1 \leqq i<j \leqq n$. As $h(A) b=0$ and $A^{k} b=b_{k}$ we have

$$
b_{2 N+n}-\delta_{1} b_{2 N+n-2}+\delta_{2} b_{2 N+n-4}-\cdots+(-1)^{N} \delta_{N} b_{n}=0 .
$$

Although this is a linear relation between the $b_{i}$ it is not a generalization of (2) as (4) for $n=2$ gives $b_{4}=\left(\alpha_{1}-\alpha_{2}\right)^{2} b_{2}$ which is weaker than (2). This suggests that (3) is not the minimum polynomial of $A$. Indeed a factor $z^{n-1}$ may be cancelled: ${ }^{2}$

TheOREM 1. Let $a, b$ be $n \times n$ matrices with elements in a field $F$. Define $b_{1}, b_{2}, \cdots$ by (1) and $\delta_{1}, \cdots, \delta_{N}$ by (3). Then

Received by the editors March 8, 1961 and, in revised form, October 10, 1961.

${ }_{1}$ The work of this author was supported by the National Science Foundation and the Deutsche Forschungsgemeinschaft.

${ }^{2}$ For a specified matrix $a$, the minimum equation of $A$ may even have a smaller degree than $2 N+1$. This leads to the problem to determine the elementary divisors of $a \times 1-1 \times a$, where $\times$ denotes the Kronecker product. 


$$
b_{2 N+1}-\delta_{1} b_{2 N-1}+\delta_{2} b_{2 N-3}-\cdots+(-1)^{N} \delta_{N} b_{1}=0 .
$$

This theorem can be easily proved by transformation of $a$ into diagonal form, if all $\alpha_{i}$ are distinct. The general case can be reduced to the one just mentioned by taking for $a$ the "generic" matrix whose $n^{2}$ elements $a_{i j}$ are independent variables. We shall not go into details as we prefer to use a more powerful method which works in arbitrary (associative) rings, not only in algebras. ${ }^{8}$ Obviously Theorem 1 is a special case $\left(R=F_{n \times n}\right)$ of

TheOREM 2. Let $R$ be $a$ ring and $\Gamma$ its center. Let $a, b \in R$ and define $b_{1}, b_{2}, \cdots$ by (1). Let a satisfy an equation $f(a)=0$ of the form

$$
f(x)=x^{n}-\gamma_{1} x^{n-1}+\gamma_{2} x^{n-2}-\cdots+(-1)^{n} \gamma_{n}, \quad \gamma_{0} \in \Gamma .
$$

Then

$$
b_{2 N+1}-\delta_{1} b_{2 N-1}+\delta_{2} b_{2 N-3}-\cdots+(-1)^{N} \delta_{N} b_{1}=0
$$

where $N=n(n-1) / 2$ and the coefficients $\delta_{k} \in \Gamma$ are defined in the following way: Let $x_{1}, \cdots, x_{n}$ be independent variables, and let $\Phi_{k}\left(c_{1}, \cdots, c_{n}\right)$ be the unique polynomial with integer coefficients which expresses the kth elementary symmetric function $d_{k}$ of the $N$ functions $\left(x_{i}-x_{j}\right)^{2}$, $1 \leqq i<j \leqq n$, in terms of the elementary symmetric functions $c_{1}^{*}, \cdots, c_{n}^{*}$ of the $x_{i}$; that is, $d_{k}=\Phi_{k}\left(c_{1}^{*}, \cdots, c_{n}^{*}\right)$. Then $\delta_{k}=\Phi_{k}\left(\gamma_{1}, \cdots, \gamma_{n}\right)$.

As the mapping $x \rightarrow a x-x a$ is a derivation in the ring $R$ one can suspect that Theorem 2 belongs to the theory of derivations and can be proved by means of that theory (cf. e.g. [2]). However, Theorem 2 can also be considered as a special case of a theorem which refers neither to derivations nor even to rings but to double modules:

TheOREM 3. Let $M$ be a left $R_{1}$-module and a right $R_{2}$-module where $R_{1}$ and $R_{2}$ are two rings over a common subring $\Gamma$ which is in the center of both $R_{1}$ and $R_{2}$. Let $\gamma x=x \gamma$ for all $\gamma \in \Gamma, x \in M$. Let $a_{1} \in R_{1}, a_{2} \in R_{2}$, $b \in M$ and define $b_{1}=a_{1} b-b a_{2}, b_{i}=a_{1} b_{i-1}-b_{i-1} a_{2}(i=2,3, \cdots)$. Let $f\left(a_{1}\right)=f\left(a_{2}\right)=0$ where $f$ is a polynomial of the form (6). Then (7) holds.

Theorem 2 is what becomes of Theorem 3 if we specialize $R_{1}=R_{\mathbf{2}}$ $=M=R$ and $a_{1}=a_{2}=a$. On the other hand, Theorem 3 is a consequence of the fact that Lagrange's resolvents work in arbitrary commutative rings, not only in fields (where they usually are employed). More specifically we need

TheOREM 4. Let $C$ be a commutative ring, and let $a^{\prime}, a^{\prime \prime} \in C$ satisfy

${ }^{3}$ We are indebted to the referee for pointing out this possibility. 
$f\left(a^{\prime}\right)=f\left(a^{\prime \prime}\right)=0$ where $f$ is a polynomial of the form

$$
f(x)=x^{n}-\gamma_{1} x^{n-1}+\gamma_{2} x^{n-2}-\cdots+(-1)^{n} \gamma_{n}, \quad \gamma_{\nu} \in C .
$$

Put

$$
g(z)=z^{2 N+1}-\delta_{1} z^{2 N-1}+\delta_{2} z^{2 N-3}-\cdots+(-1)^{N} \delta_{N} z
$$

where $\delta_{k}=\Phi_{k}\left(\gamma_{1}, \cdots, \gamma_{n}\right), k=1, \cdots, N$, is defined as in Theorem 2. Then $g\left(a^{\prime}-a^{\prime \prime}\right)=0$.

Theorem 3 follows from Theorem 4 if we choose for $a^{\prime}$ the endomorphism $x \rightarrow a_{1} x$ of $M$, for $a^{\prime \prime}$ the endomorphism $x \rightarrow x a_{2}$ of $M$, and for $C$ that subring of the endomorphism ring of $M$ which is generated by $a^{\prime}, a^{\prime \prime}$ and the $n$ endomorphisms $x \rightarrow \gamma_{\nu} x=x \gamma_{\nu}$.

We prove Theorem 4 by means of an elementary algebraic identity:

LeMma. Let $x, y, z, c_{1}, c_{2}, \cdots, c_{n}$ be independent variables over the field $Q$ of rational numbers, and let $Z$ denote the ring of rational integers. Put

$$
f(x)=f\left(x ; c_{1}, \cdots, c_{n}\right)=x^{n}-c_{1} x^{n-1}+c_{2} x^{n-2}-\cdots+(-1)^{n} c_{n}
$$

and

$$
g(z)=g\left(z ; c_{1}, \cdots, c_{n}\right)=z^{2 N+1}-\Phi_{1} z^{2 N-1}+\cdots+(-1)^{N} \Phi_{N} z
$$

where $\Phi_{k} \in Z\left[c_{1}, \cdots, c_{n}\right], k=1, \cdots, N$, is defined as in Theorem 2. Then there exist polynomials $p, q \in Z\left[x, y, c_{1}, \cdots, c_{n}\right]$ such that

$$
g(x-y)=p f(x)+q f(y)
$$

identically in $x, y, c_{1}, \cdots, c_{n}$.

In order to prove the lemma we divide $g(x-y)$, qua polynomial in $x$, by $f(x)$. As the highest coefficient in $f(x)$ is 1 no denominators arise, and we obtain

$$
g(x-y)=p f(x)+\sum_{\mu=1}^{n-1} r_{\mu} x^{\mu}
$$

where $p \in Z\left[x, y, c_{1}, \cdots, c_{n}\right]$ and $r_{\mu} \in Z\left[y, c_{1}, \cdots, c_{n}\right]$. Now we divide each $r_{\mu}$, qua polynomial in $y$, by $f(y)$ and obtain

$$
g(x-y)=p f(x)+q f(y)+\sum_{\mu, \nu}^{n-1} r_{\mu \nu} x^{\mu} y^{\prime}
$$

where $q \in Z\left[x, y, c_{1}, \cdots, c_{n}\right]$ and $r_{\mu \nu} \in Z\left[c_{1}, \cdots, c_{n}\right]$. In order to prove $r_{\mu \nu}=0$ we take independent variables $x_{1}, \cdots, x_{n}$ and denote 
their elementary symmetric functions by $c_{1}^{*}, \cdots, c_{n}^{*}$. By definition of $g(z)$ we have (cf. (3))

$$
g\left(z ; c_{1}^{*}, \cdots, c_{n}^{*}\right)=z \prod_{i \neq j}\left[z-\left(x_{i}-x_{j}\right)\right]
$$

hence

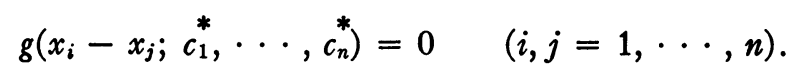

This together with (9) shows that the polynomial

$$
r^{*}(x, y)=\sum_{\mu, \nu}^{n-1} r_{\mu \nu}^{*} x^{\mu} y^{\nu}, \quad r_{\mu \nu}^{*}=r_{\mu \nu}\left(c_{1}^{*}, \cdots, c_{n}^{*}\right)
$$

has the $n^{2}$ zeros $x=x_{i}, y=x_{j}$ in the field $Q\left(x_{1}, \cdots, x_{n}\right)$. As the degrees of $r^{*}$ with respect to $x$ and $y$ are both less than $n$ we find $r_{\mu \nu}^{*}=0$, hence $r_{\mu \nu}=0$ as $c_{1}^{*}, \cdots, c_{n}^{*}$ are algebraically independent. Hence (9) reduces to (8), and the lemma is proved. Theorem 4 follows from the lemma by specializing $x=a^{\prime}, y=a^{\prime \prime}$ and $c_{i}=\gamma_{i}$ in (8).

\section{REFERENCES}

1. T. Kato and O. Taussky, Commutators of $A$ and $A^{*}$, J. Washington Acad. Sci. 46 (1956), 38-40.

2. S. A. Amitsur, Derivations in simple rings, Proc. London Math. Soc. 7 (1957), 87-112.

3. C. C. MacDuffee, The theory of matrices, Springer, Berlin, 1933; pp. 83-84, 89.

California Institute of Technology

University OF TüBINGEN, Germany 